\title{
Die Freiheit des Willens - ein noch aktueller Begriff?
}

Von Godehard Brüntrup, Hochschule für Philosophie München

Die Möglichkeit der Willensfreiheit wird vor allem von der Seite der empirischen Wissenschaften oft negiert. Über die bekannten philosophischen Probleme hinaus ${ }^{1}$ ist Freiheit somit in jüngster Vergangenheit zu einem prekären Begriff geworden. Die Freiheit des Willens scheint aber eine unaufgebbare Grundlage unseres Rechtssystems zu sein. Damit einer Person eine Tat überhaupt als Handlung zugerechnet werden kann, muss eine willentliche Kontrolle der Tatausführung gegeben sein. Wenn jemand keinerlei willentliche Kontrolle über die Handlung hat, dann handelt es sich jedenfalls in einem engeren Sinne gar nicht mehr um eine moralisch bewertbare Handlung, sondern einfach um eine Körperbewegung. Steht jemand beispielsweise unter einer derart starken Form der Hypnose, dass die vermeintlichen Handlungen vollständig von einer anderen Person kontrolliert werden, so ist die „handelnde“ Person auf den Status einer „Marionette“ gesunken. Ein anderer Akteur zieht die Fäden, übt die vollständige Kontrolle aus und verantwortet daher die Handlungen. Die Fähigkeit zur Selbstkontrolle ist also ein notwendiges, wenn auch nicht hinreichendes Element von Freiheit. Der Mangel an Selbstkontrolle kann äußerlich oder innerlich verursacht sein. Unter anderem wegen einer eingeschränkten Impulskontrolle, spricht man Kindern die Mündigkeit ab. Sie sind ihren eigenen Antrieben ausgeliefert. Aber auch ein Erwachsener mit voll ausgeprägter Meta-Kontrolle der eigenen Bedürfnisse und Affekte ist nicht für eine Tat verantwortlich zu machen, wenn sie für ihn durch äußeren Zwang alternativlos ist.

Neben der willentlichen Kontrolle wird normalerweise ein hinreichender Vernunftgebrauch als konstitutiv für die Ausübung von juristisch relevanter Freiheit vorausgesetzt. Hierzu gehört einerseits die Fähigkeit, das moralisch Gute vom moralisch Bösen zu unterscheiden,

\footnotetext{
${ }^{1}$ Vgl. Keil, Geert. Willensfreiheit. Berlin: DeGruyter (2017).
} 
andererseits die Fähigkeit, die möglichen Folgen einer Handlung wenigstens prinzipiell verstehen zu können. Der Zusammenhang besteht deshalb, da die ethische Bewertung einer Handlung in den allermeisten Fällen die Fähigkeit voraussetzt, die Folgen der Handlung erfassen zu können. Die Fähigkeit zur kognitiven Evaluation einer Handlung und deren Folgen ist daher ebenso ein notwendiges, wenn auch nicht hinreichendes Element von Freiheit.

In gewisser Weise ist damit schon das Wesentliche gesagt. Die Kriterien freien Handelns, die im juristischen Kontext relevant sind, lassen sich in den Grundzügen aus dem gesunden Menschenverstand ableiten. Warum sollte man für die juristische Praxis noch tiefere Fragen stellen? Zunächst ist das Thema für eine philosophische Analyse gar nicht wirklich einladend, da wir gar nicht vor einem Rätsel stehen, das die Anstrengung des philosophischen Begriffs provozieren würde. Erst der aktuelle Angriff auf den Freiheitsbegriff in der öffentlichen Debatte macht den Rekurs auf eine philosophische Anstrengung des Begriffs nötig. Die sich aus den Anfragen der Hirnforschung ergebenden Herausforderungen lassen sich nur durch eine philosophische Analyse meistern.

\section{Freiheit und Determinismus}

Daher müssen wir, bevor wir zu der empirischen Forschung kommen, ein philosophisches Instrumentarium erarbeiten. Das Zentrum der relevanten philosophischen Debatte ist die Verhältnisbestimmung von Freiheit und Determinismus. Wenn unsere Welt deterministisch ist, dann gibt es zu jedem beliebigen Zeitpunkt nur eine mögliche Zukunft. Die Vergangenheit zusammen mit den Naturgesetzen legt genau eine mögliche Zukunft fest. Strikt genommen folgt also aus dem initialen Zustand des Universums beim Urknall plus den Naturgesetzen alles andere mit Notwendigkeit. In einer solchen Welt gibt es keine offene Zukunft, alle Entscheidungen sind bereits getroffen, es gibt keinen Spielraum, der nicht durch die Vergangenheit und die Naturgesetze bereits vollständig ausgefüllt wäre. In einer solchen Welt wäre dann prima facie kein Raum für Freiheit, weil niemand für etwas verantwortlich gemacht werden kann, über das er keine Kontrolle hat. Niemand von uns hat Kontrolle darüber, was sich in der weit entfernten Vergangenheit, sagen wir vor 10 Millionen Jahren, ereignete. Niemand von uns hat Kontrolle über die Naturgesetze in dem Sinne, dass er sie selbst oder deren Geltungsbereich ändern könne. Wenn nun aber die Gegenwart mit 
Notwendigkeit aus der weit entfernten Vergangenheit und den Naturgesetzen folgt, dann hat niemand Kontrolle darüber, was sich in der Gegenwart ereignet. Insbesondere hat dann niemand Kontrolle über seine eigenen Handlungen. In diesem Sinne kann man sagen, dass Notwendigkeit und Freiheit sich gegenseitig ausschließen. In einer deterministischen Welt gibt es keine Freiheit.

In dieser Situation kann man sich fragen, ob es weiterführen würde, den Zufall in die Überlegungen einzubeziehen. Einige Interpretationen der Quantenmechanik implizieren, dass sich einige Ereignisse in der physischen Welt ohne zureichenden physischen Grund ereignen. Es handelt sich also um zufällige, aus der Vergangenheit nicht ableitbare Ereignisse. Der Verlauf einer solchen Welt ist dann nicht mehr deterministisch, weil es in ihr mehr als einen möglichen Zukunftsverlauf gibt.

Die Einführung des Indeterminismus hilft uns aber für das Problem der Freiheit nicht unmittelbar weiter. Ein zufälliges Ereignis ist ja eines, das grundlos geschehen ist. Nehmen wir an, man könnte die Zeit beliebig oft zurückdrehen und wie ein Videoband unverändert neu ablaufen lassen. In einer indeterministischen Welt könnte auf die identische Vergangenheit mehr als eine mögliche Zukunft folgen. Nehmen wir weiter an, dass sich eine handelnde Person mit einer anstehenden Entscheidung große Mühe gibt und alle Vor- und Nachteile gewissenhaft abwägt. Nachdem dieser Abwägungsprozess abgeschlossen ist, halten wir die Uhr an und spulen die Zeit zurück. Wenn wir dies beispielsweise $100 \mathrm{Mal}$ täten, so würde in einem gewissen Prozentsatz der Fälle die Person eine bestimmte Handlung A vollziehen und in einem anderen Prozentsatz eine andere Handlung B vollziehen. Die Vergangenheit und der Abwägungsprozess, der in der Vergangenheit enthalten ist, legt die Zukunft nicht eindeutig fest. Ist dann die Handlung überhaupt noch Resultat des Abwägungsprozesses? Wenn sie das nicht ist, muss sie dann nicht von der handelnden Person als „fremd“ und außerhalb ihrer Kontrolle erlebt werden? Welche Handlung ausgeführt wird, ist dann nämlich im Rahmen von bestimmten Wahrscheinlichkeiten eine Sache des reinen Zufalls. Niemand hat Kontrolle über einen Zufall, niemand kann für einen Zufall verantwortlich gemacht werden. In einer indeterministischen Welt kann es daher keine Freiheit geben.

Wir sind nun in ein wirklich schwerwiegendes Dilemma geraten. Die Welt ist entweder deterministisch oder sie ist indeterministisch. Zu dieser Alternative gibt es kein Drittes. Wir haben aber gesehen, dass sowohl im Determinismus wie auch im Indeterminismus Freiheit 
unmöglich zu sein scheint. Beide Hörner des Dilemmas führen uns also geradewegs in eine Welt, in der es Freiheit gar nicht geben kann. Im Verlaufe der Geschichte der Philosophie wurden jedoch Theorien entwickelt, die es erlauben, in beiden möglichen Alternativen Konzeptionen von Willensfreiheit zu verteidigen. Dasjenige Konzept, welches Freiheit und Determinismus für miteinander verträglich hält, nennt man dementsprechend „Kompatibilismus“. Dasjenige, welches Freiheit nur in einer indeterministischen Welt für möglich hält, nennt man „Libertarismus“.

\section{Kompatibilismus}

Beginnen wir mit dem ersten Horn des Dilemmas, also der Annahme, dass sich unsere Welt komplett deterministisch verhalte. Unser Gehirn wird von vielen als ein deterministisches System betrachtet. Wir Menschen sind nach dieser Auffassung als mittelgroße physische Systeme nicht anfällig für einen möglichen Indeterminismus auf der Mikroebene, so dass sich dann das Gehirn nicht von quantenmechanischen Unbestimmtheiten beeinflussen lässt. Es soll vollständig als deterministische neuronale Maschinerie begriffen werden. Wenn nun, das sei einmal angenommen, das Gehirn die bestimmende materielle Grundlage unseres Denkens sei, dann folgt, dass auch unsere Denkprozesse deterministisch ablaufen. Das bedeutet in Bezug auf das bereits erörterte Beispiel: Wenn die betreffende Person eine mögliche Entscheidung von allen Seiten her beleuchtet und abwägt und sich dann aufgrund dieser Erwägungen in der Vergangenheit für eine bestimmte Handlung entschließt und diese durchführt, dann wird derselbe Prozess des Überlegens immer wieder zur exakt selben Handlung führen. Wenn also die Geschichte zurückgespult würde und genau identisch wieder abliefe, so würde die Person immer wieder in exakt gleicher Weise handeln. Hier tritt nun etwas Überraschendes auf. Wir empfinden diese Determination des eigenen Handelns in gewisser Weise gar nicht mehr als Bedrohung unserer Freiheit, sondern als Ermöglichungsgrund selbstbestimmten Handelns. Wenn nach meiner ausführlichen Überlegung einmal die eine und ein anderes Mal eine andere Handlung folgen könnte, so erlebte ich mich gar nicht mehr als Autor und Ursprung dieser Handlungen. Gemäß diesem Gedankengang ist also alternativlose Determination ein Ermöglichungsgrund von Selbstbestimmung und Freiheit. Determination sichert die Selbstbestimmung. So lange also eine Handlung aus den eigenen Überlegungen notwendig folgt, scheint Notwendigkeit nicht 
in einen Konflikt mit Freiheit zu führen. Unfreiheit käme erst dann ins Spiel, wenn es nicht meine eigenen Überlegungen wären, die die Ursache meiner Handlungen sind, sondern Einflüsse, die von außen kommen. Wenn also beispielsweise jemand mich unter Androhung von Gewalt zu einer Handlung zwänge, dann läge offensichtlich keine Selbstbestimmung und keine Freiheit vor. Im kompatibilistischen Verständnis ist es also nicht der Determinismus, der die Freiheit bedroht, sondern Freiheit ist dadurch bedroht, dass es Hindernisse gibt, die mich an der Durchsetzung meines eigenen Willens hindern. Wenn ich den Wunsch habe, den Raum zu verlassen und werde daran mit Gewalt gehindert, so liegt ein klarer Fall von Unfreiheit vor.

Nehmen wir nun einmal hypothetisch an, unsere gesamte Welt befände sich im Zwangskorsett des Determinismus. Es gäbe dann zu jedem Zeitpunkt genau eine mögliche Zukunft. Selbst in einigen Interpretationen der Quantenmechanik ist dies der Fall. Wir wissen nicht mit letzter Sicherheit, ob unsere Welt deterministisch oder indeterministisch ist. Wäre unsere Welt also deterministisch, dann könnten wir immer noch ohne Schwierigkeit die relevanten Unterscheidungen treffen: Fälle, in denen Menschen ihren Willen ungestört und ohne Zwang durchsetzen können, könnten wir klar absetzen von denen, in denen Menschen aufgrund äußeren Zwanges diese Möglichkeit genommen ist. Im ersten Falle sind die betroffenen Personen frei, im zweiten Falle wurden sie ihrer Freiheit beraubt. In diesem Sinne wäre auch in einer deterministischen Welt die Möglichkeit gegeben, die juristisch relevante Unterscheidung von Freiheit und Zwang zu erhalten. Die Abwesenheit von äußerem Zwang ist vielleicht ein minimalistisches Kriterium für Freiheit, aber eines, das für viele Beispiele aus der juristischen Praxis bereits hinreichend ist. Es wird sich in denjenigen Fällen als nicht hinreichend erweisen, wo wir von einem inneren Zwang sprechen. Ein innerer Zwang ist dann gegeben, wenn ich einen Wunsch, der in mir aufsteigt, als mir wesensfremd oder als fremdbestimmt erlebe und gleichzeitig nicht die Möglichkeit habe, diesen Wunsch durch eine willentliche Anstrengung so zu unterdrücken, dass er nicht mehr handlungsauslösend wird. In diesem Fall handelt es sich um eine innere Versklavung, also um die Unmöglichkeit, die wirklichen Absichten und Intentionen gegen entgegenlaufende psychische Kräfte durchzusetzen. Hier ist also eine Form von Kontrollverlust gegeben.

Ebenso wie das Kleinkind keine Kontrolle über plötzlich in ihm aufsteigende Bedürfnisse hat und deshalb auch nicht als strafmündig erachtet wird, so hat der zwanghaft handelnde Erwachsene keine ausreichende Kontrolle über die eine Handlung auslösenden mentalen 
Mechanismen. Er muss Ihnen folgen, auch gegen bessere Einsicht und gegen einen zu schwachen widerstrebenden Willen.

Hiermit wäre eine zweite Form der Unfreiheit gegeben, die ebenfalls dadurch definiert ist, dass man seinen eigenen Willen nicht in Handlungen umsetzen kann. Das Hindernis, welches die Ausführung der Handlung verhindert, liegt nun aber nicht außerhalb der handelnden Personen, sondern es resultiert aus Strebungen in der eigenen Psyche, über die die handelnde Person keine Kontrolle hat. Auch in einer deterministischen Welt könnte man durch entsprechende Testverfahren feststellen, ob jemand unter Zwangsstörungen der beschriebenen Art leidet, die seinen Freiheitsraum eingrenzen. Auch in einer vollkommen deterministischen Welt könnten wir also unterscheiden, ob innere mentale Freiheit vorliegt oder nicht. Wenn wir also Freiheit ganz minimalistisch definieren als die Fähigkeit, meine Wünsche in die Tat umsetzen zu können, dann scheint der Determinismus keine Bedrohung für die Freiheit darzustellen. Auch wenn die neuronalen Prozesse in unseren Gehirnen also vollständig deterministisch abliefen, wäre das dann kein Einwand gegen einen derart bestimmten Freiheitsbegriff.

\section{Libertarismus}

Dieser minimalistische Freiheitsbegriff des Kompatibilismus ist aber nicht ganz unproblematisch. Weil er mit einer deterministischen Welt kompatibel ist, erlaubt er Freiheit auch dann noch, wenn die handelnde Person gar keine echten Alternativen hat. Ist das ein Problem? Es gibt Fälle, wo wir jemanden für eine Handlung verantwortlich machen, obwohl der betreffenden Personen letztendlich keine Handlungsalternativen zur Verfügung standen. Nehmen wir an, eine Person verfügte nur über die Alternative entweder die blaue oder die rote Pille zu schlucken. Der Person wird mitgeteilt, dass sich in der roten Pille ein bestimmter psychoaktiver Wirkstoff befindet, während sich in der blauen Pille nur Vitamine befinden. In Wirklichkeit befindet sich aber in beiden Pillen die angegebene psychoaktive Substanz. Wenn sich nun eine Person für die rote Pille entscheidet, von der sie glaubt, dass sie eine psychoaktive Substanz enthalte, dann können wir sie für ihren Rausch verantwortlich machen, obwohl sie gar keine Alternative hatte. Hätte sie nämlich die blaue Pille gewählt, so hätte das zum selben Resultat geführt. Wenn es also möglich ist, dass jemand ohne echte Handlungsalternativen immer noch für seine Handlungen verantwortlich gemacht werden 
kann, dann scheint auch in einer deterministischen Welt Verantwortung möglich zu sein. Hier muss man allerdings genauer hinschauen. In unserem Beispiel hatte die handelnde Person ja zumindest die Freiheit, entweder die blaue oder die rote Pille zu nehmen. Diese Handlungsalternative bestand, auch wenn die Folgen beider Handlungen schlussendlich identisch sind. Aber in dieser Entscheidung gab es doch eine innere Wahl unter zwei klar definierten Alternativen. Nur weil es dieses kurze Aufflackern einer selbstbestimmten Alternative gab, schreiben wir der Person echte Verantwortung zu.

Hätte es nämlich seit tausend Jahren festgestanden, dass die Person die blaue Pille nehmen würde, so müsste man folgern, dass die Person nicht wirklich verantwortlich ist für Ihre Entscheidung, die blaue Pille zu nehmen. Die Entscheidung, welche Pille sie nehmen würde, war schon gefallen, lange bevor diese Person überhaupt existierte. Dies ist eine Konsequenz des Determinismus. Aus diesem Grunde optieren manche Philosophinnen und Philosophen für das zweite Horn unseres Dilemmas: den Libertarismus. Libertarier behaupten, dass es Freiheit nur in einer indeterministischen Welt geben kann. Welchen Vorteil soll aber die Einführung des Indeterminismus bringen? Die Kausalkette in die Vergangenheit würde nicht mehr stark genug sein, um alle Ereignisse aus der weit entfernten Vergangenheit herzuleiten. Selbst innerhalb der Lebensgeschichte der handelnden Personen wäre es nicht mehr der Fall, dass ihr jetziges Handeln vollständig von ihren früheren geistigen und körperlichen Zuständen determiniert wäre. Damit tut sich aber das oben erwähnte Problem auf. Wenn auf exakt dieselbe Geschichte des Abwägens zwei unterschiedliche Handlungen folgen können, dann scheint es so zu sein, dass die handelnde Person gar keine Kontrolle darüber besitzt, welche der beiden Handlungen sie am Ende vollzieht. Der Zufall scheint also die Freiheit zu zerstören.

Diese Konsequenz ist aber nicht zwingend. Es gehört nicht wesentlich zu einer freien Handlung, dass sie mit Notwendigkeit aus der Vergangenheit folgt. Im Gegenteil: Ein wirklich freier Wille darf durch kein vorheriges Ereignis determiniert sein. Nicht einmal die eigenen mentalen Zustände kurz vor dem freien Willensentschluss sollen den freien Willen vollständig determinieren. Daraus folgt nicht, dass die Handlung dann grundlos erfolgt. Wenn die handelnde Person zwei Alternativen rational abwägt und sich dann für eine entscheidet, so handelt sie im Lichte von Gründen. Jede der beiden Alternativen kann gute Gründe für sich ins Feld führen. Wenn ich mich für eine der beiden Begründungen entscheide, so ist dies eben eine begründete Entscheidung. Die Willensentscheidung erfolgt 
in einem rationalen Kontext. Die handelnde Person kann für ihre Handlung Gründe angeben. Die Aneignung und Bejahung der Gründe liegt also nicht vor der Entscheidung für eine Handlung, sondern die Gründe werden gleichzeitig mit der Entscheidung ergriffen und bejaht. Es ist eine Entscheidung für die im Moment als besser bewerteten Gründe. Für diese Entscheidung muss es keine weiteren Gründe geben. Welche Gründe letztendlich für die besseren gehalten werden, lässt sich nicht vollständig aus dem vorhergehenden Abwägungsprozess ableiten. Dies ist das Moment der freien Selbstbestimmung im Lichte von Gründen, das aus der Vergangenheit nicht ableitbar ist. Wenn also zwischen zwei alternativen Handlungsbegründungen entschieden wird, so gibt es nicht noch einmal Gründe für die Gründe. Sonst könnte man in einer unendlichen Kette immer weiter nach den Gründen für die Gründe der Gründe fragen. Die Handlung, die aus einer solchen freien Willensentscheidung resultiert, ist daher nicht unvernünftig. Es ist eine Handlung aus Gründen. Welche Gründe aber eine handelnde Person für die handlungsrelevanten hält, kann man nicht vollständig aus früheren mentalen Zuständen der Person ableiten.

Nach der Auffassung des Libertarismus wird also in der freien Handlung ein radikaler Anfang gesetzt, der als spontane Selbstbestimmung charakterisiert werden könnte. In der Tat hat eine solche Entscheidung in einem spezifischen Sinn ein Moment des Zufälligen. Zufällig ist die freie Handlung deshalb, weil sie sich nicht vollständig aus der Vergangenheit herleiten lässt. Aus der Innenperspektive der handelnden Person ist genau dies aber eine radikale Selbstbestimmung in der Gegenwart. Selbst die eigene psychische Vergangenheit determiniert eine Person nicht vollständig in ihrem Handeln. Jeder Moment im selbstbestimmten Leben der handelnden Personen hat also einen Aspekt des Unverfügbaren, der sich nicht aus der Kette der Kausalverbindungen ableiten lässt. Dieses unverfügbare Moment der Entscheidung ist aber nicht irrational und damit gerade nicht bloß zufällig, sondern es ist das sich selbst bestimmende Ergreifen von Gründen. Das ist die Kernthese des Libertarismus. Die Kompatibilisten, die Freiheit mit Determinismus zusammen denken wollen, sehen in diesem Element der aus der Vergangenheit unableitbaren Entscheidung eine Gefahr für die Freiheit (Zufall). Diejenigen, die Freiheit und Determinismus für unvereinbar halten, sehen umgekehrt gerade in der vollständigen Bestimmung des Willens durch frühere mentale Zustände eine Gefahr für die Willensfreiheit (Notwendigkeit). 


\section{Ein pragmatischer Pluralismus}

Die beiden vorgestellten Konzeptionen der Willensfreiheit werden in der Philosophie seit Jahrhunderten diskutiert, ohne dass eine allseits akzeptierte Entscheidung für eine der beiden Alternativen in Sicht wäre. Man kann auch die beiden Theorien nicht einfach integrieren, denn offensichtlich unterscheiden sich beide Konzeptionen in wesentlichen Punkten. Der Kompatibilismus bestimmt Freiheit negativ als die Abwesenheit von äußerem und innerem Zwang, der Libertarismus bestimmt Freiheit positiv als die selbst gewählte Wahl zwischen vorher nicht fixierten Alternativen. Beide legen mit nachvollziehbaren Argumenten ein stimmiges Gesamtbild vor, in welchem vermieden werden kann, dass die Möglichkeit von Freiheit zwischen Determination und Zufall zerrieben wird. Damit ist ein wichtiger Punkt in der Argumentation erreicht. Das oben dargelegte Dilemma der Freiheit muss nicht dazu führen, dass man den Freiheitsbegriff ganz aufgibt.

Glücklicherweise ist es für viele pragmatische Kontexte gar nicht notwendig, für einen der beiden Freiheitsbegriffe Partei zu ergreifen. Beide Konzeptionen können beispielsweise als philosophische Grundlage der gängigen Rechtsprechung herangezogen werden, da beide Freiheitsbegriffe in der Praxis eine Unterscheidung zwischen freien und unfreien Handlungen erlauben. Der stärkere positive Freiheitsbegriff des Libertarismus kann die negativen Freiheitskriterien des Kompatibilismus nämlich problemlos integrieren. Ob eine Handlung frei ist, können wir empirisch nur dadurch begründen, dass wir die Abwesenheit von äußerem und innerem Zwang konstatieren. Darin kommen sowohl Kompatibilismus wie auch Libertarismus überein. Ob darüber hinaus noch jene Möglichkeit der durch die Vergangenheit nicht festgelegten Selbstbestimmung besteht, die der Libertarismus fordert, können wir nicht mit Sicherheit sagen. Es handelt sich um eine metaphysische These, die aber unseren besten Theorien über die Natur nicht offensichtlich widerspricht. Es ist nicht vollkommen ausgeschlossen, dass sich quantenmechanische Indeterminismen auf das Gehirn als nichtlineares System auswirken können. Manche Wissenschaftler argumentieren für einen Indeterminismus im Verhalten von Lebewesen. ${ }^{2}$ Vertreter des Kompatibilismus werden nun argumentieren, dass wir diese Spekulationen über den Zusammenhang von Freiheit und einer offenen Zukunft gar nicht benötigen. Vertreterinnen des Libertarismus

\footnotetext{
${ }^{2}$ Brembs, Björn. "Towards a scientific concept of free will as a biological trait: spontaneous actions and decision-making in invertebrates." Proc. R. Soc. B (2011): 278, 930-939.
} 
werden antworten, dass sich nur in dieser Konzeption eine gehaltvolle Idee der moralischen Verantwortung begründen lässt. Nur wenn jemand wirklich über Alternativen verfügt, die nicht von der weit entfernten Vergangenheit vorherbestimmt wurden, kann man diese Person wirklich für ihr Handeln verantwortlich machen. Dieser starke Begriff der moralischen Verantwortung ist aber für die juristische Einschätzung, ob eine Tat frei oder unfrei vollzogen wurde, gar nicht notwendig. Hier kann es durchaus genügen, Freiheit des Willens dann anzunehmen, wenn eine Person des Vernunftgebrauches fähig ist und weder durch äußeren noch durch inneren Zwang daran gehindert wird, ihre Entscheidungen in die Tat umzusetzen. Moralische Verantwortung in diesem schwächeren Sinne kann einer Person also auch in einer deterministischen Welt zugesprochen werden. Auch in einer deterministischen Welt können wir Personen, die jene Kriterien erfüllen, unterscheiden von denjenigen, die sie nicht erfüllen. Da in einer deterministischen Welt auch diejenigen Personen, die über Vernunftgebrauch verfügen und nicht unter äußeren oder inneren Zwang stehen, keine wirklichen Alternativen haben, bleibt hier allerdings ein gewisses Unbehagen zurück. Kann man wirklich sagen, dass jemand, dessen Handlungen von den Naturgesetzen und dem Zustand der Welt in der weit entfernten Vergangenheit determiniert sind, moralische Verantwortung trägt? Die kompatibilistische Konzeption wird Strafe daher eher als eine Maßnahme zur Verhaltensmodifikation und nicht als Sühne verständlich machen können. Für die libertarische Konzeption ist es hingegen leicht, die Handlung ganz und gar der Person zuzuschreiben, weil es sich ja laut der Theorie um eine spontane Selbstsetzung handelt. Da diese spontane Selbstsetzung aber das Moment des Zufälligen nicht ganz abschütteln kann, gerät man auch hier wieder in die Nähe einer Pattsituation. Keine der beiden Theorien kann entscheidende Überlegenheit für sich verbuchen. Wahrscheinlich ist es so, dass eine restlos befriedigende philosophische Explikation des Freiheitsbegriffes nicht möglich ist. Jede der beiden großen Alternativen hat ihre theoretischen Defizite. Es bleibt aber dabei, dass beide Freiheitsbegriffe als Grundlage unserer Praxis bei der Zuschreibung von Verantwortung genutzt werden können. Es kann also konstatiert werden, dass wir für die Bewertung menschlicher Praxis über zwei praktikable Freiheitsbegriffe verfügen, die dem oben entwickelten Dilemma zwischen Zufall und Notwendigkeit entrinnen. Es ist daher auf den ersten Blick gar nicht nachzuvollziehen, warum sich in jüngster Zeit eine Debatte darüber entwickelt hat, ob der Freiheitsbegriff überhaupt noch zeitgemäß sei. Hier wird unterstellt, dass der Freiheitsbegriff für aufgeklärte und 
wissenschaftlich gebildete Menschen nicht mehr zu halten sei. Wir haben aber gesehen, dass diese These rein philosophisch nicht überzeugend ist. Nach unserer Auffassung liegt der wahre Grund für die Popularität dieser These anderswo.

\section{Freiheit und Gehirn}

Freiheit hat durch popularisierte Ergebnisse der empirischen Forschung einen schlechten Ruf bekommen. Die Gebildeten unter ihren Leugnern verweisen gerne auf psychologische und neurophysiologische Studien. All diese Studien zeigen auf, dass viele Handlungen, von denen wir glauben, dass sie unter unserer bewussten Kontrolle stehen, in Wirklichkeit von unbewussten Instanzen beherrscht werden. Die These, dass nur dasjenige frei getan werden könne, was unter bewusster Kontrolle stehe, ist aber begründungsbedürftig. Wenn zum Beispiel eine Läuferin beim Startschuss automatisch und ohne bewusste Entscheidung losläuft, dann folgt daraus nicht unmittelbar, dass sie unfreiwillig am Lauf teilgenommen hätte. Wir wissen spätestens seit Freud, dass nur ein geringer Teil unseres psychischen Geschehens in das aufmerksame Wachbewusstsein eintritt und willentlicher Kontrolle unterliegt. Folgt daraus aber, dass wir der Freiheit beraubt sind? Ein Beispiel: Kaum jemand vollzieht beim Autofahren jede Handlung des Schaltens und des Lenkens mit bewusster Aufmerksamkeit, vieles läuft automatisch ab. Erst dann, wenn Schwierigkeiten auftreten, richtet man seine Aufmerksamkeit bewusst auf das Schalten, Bremsen oder Lenken. Wenn das Bewusstsein sich einschaltet, dann kann eine unbewusst begonnene Tätigkeit unterbrochen und kontrolliert werden. Aus der Tatsache, dass eine Autofahrt zu erheblichen Teilen aus solchen unbewussten Tätigkeiten besteht, würde niemand folgern, dass sie unfrei und moralisch nicht zurechenbar sei. Um die Willensfreiheit zu widerlegen, müsste man also zeigen, dass der Abwägungsprozess zwischen den Gründen, die für oder gegen die Reise sprechen, gar nicht unter der bewussten Kontrolle der handelnden Person steht. Wenn also auch unbewusst vollzogene Körperbewegungen freie Handlungen sein können, gibt es dann überhaupt einen Zusammenhang zwischen Freiheit und Bewusstsein? In der Tat ist es so, dass wir einer bewusstseinslosen Maschine Freiheit normalerweise nicht zugestehen. Der Grund dafür liegt darin, dass wir einen Zusammenhang zwischen Freiheit, bewusster Aufmerksamkeit und Absichtlichkeit feststellen. Eine Handlung, die unabsichtlich ausgeführt wird, ist in einem spezifischen Sinne weniger frei. Sie ist aber nicht 
notwendigerweise völlig unfrei. Das erkennt man daran, dass wir Menschen für unabsichtliche Handlungen durchaus moralisch verantwortlich machen. Wenn beispielsweise ein übermüdeter Apotheker unabsichtlich das falsche Medikament ausgibt, so entbindet ihn das nicht von der Verantwortung. Dies liegt aber darin begründet, dass er in diesem Zustand seinen Dienst nicht hätte ausüben dürfen. Es gibt also eine bewusste Entscheidung im Hintergrund, die ihn in die moralische Pflicht nimmt. Die unbewusste Entscheidung ist also in den Kontext einer umfassenderen bewussten Entscheidung eingebettet.

Wenn aber gar kein Kontext bewusster Entscheidung vorliegt, dann fehlt jene Art der mentalen Repräsentation, die für eine absichtliche Körperbewegung notwendig ist. Es gibt einen tiefen Zusammenhang zwischen dem Bewusstsein und dem Phänomen der Intentionalität, d.h. der Fähigkeit, etwas geistig zu repräsentieren. Nur ein bewusster mentaler Gehalt kann der Gegenstand einer Absicht sein. Daher können wir es uns nicht vorstellen, dass völlig bewusstseinslose Wesen etwas geistig so repräsentieren können, dass sie es auch beabsichtigen. Folgt aus dieser Einsicht aber, dass jede Handlung, die von einem unbewussten Prozess ausgelöst wurde, unfrei ist? Dies ist dann offensichtlich nicht der Fall, wenn der unbewusste Prozess in einen größeren vom Bewusstsein gesteuerten Zusammenhang eingebettet ist. Dies ist zum Beispiel im Falle der Läuferin gegeben. Sie hat sich bewusst unter Abwägung von Gründen entschieden, an diesem Wettkampf teilzunehmen. Hier kann es durchaus eine langwierige Abwägung zwischen Alternativen gegeben haben. Der übergeordnete Grund mag beispielsweise darin liegen, die Qualifikation für die Olympischen Spiele zu erreichen. Die eigentliche Handlung des Laufens kann aber durch einen automatisierten, reflexhaften Prozess ausgelöst werden. Würde hier wieder eine bewusste Entscheidung gefordert, so wäre der zeitliche Aufwand kontraproduktiv hoch. Trotzdem würde niemand auf die Idee kommen zu behaupten, die Teilnahme an dem Lauf sei unfrei. 


\section{Freiheit empirisch widerlegt?}

Mit diesen begrifflichen Unterscheidungen im Hintergrund kann man nun die viel diskutierten Experimente, die unsere Unfreiheit belegen sollen, genauer analysieren. In dem berühmten Experiment von Benjamin Libet ${ }^{3}$ werden Menschen dazu aufgefordert, in den nächsten 20 Sekunden spontan einen Finger zu heben. Dabei sollen sie eine sehr genaue Uhr betrachten und feststellen, wann ihnen bewusst wurde, dass sie den Finger heben werden. Er stellt sich nun heraus, dass sich im Durchschnitt der Versuche einen Sekundenbruchteil vor dem bewussten Entschluss, den Finger zu heben, bereits ein unbewusstes Potenzial im Gehirn aufbaut, das allein ausreicht, die Bewegung auszulösen. Das Bewusstsein kommt also zu spät, um die Handlung einzuleiten.

Die Verlässlichkeit solcher Messungen ist nicht unumstritten. Denn es gilt, die äußere intersubjektiv gemessene Zeit mit einer subjektiven Datierung eines innerpsychischen Prozesses zu korrelieren. Ob dies mit der hinreichenden Exaktheit gelingen kann, darf man hinterfragen. Wie können wir eine Gleichzeitigkeit oder ein Früher oder Später feststellen, wenn dem externen Beobachter die innere Perspektive der Versuchsperson nicht zugänglich ist? Er muss sich auf einen Report der Versuchsperson verlassen. In unserem Experiment schauten die Teilnehmer auf eine sehr genaue Uhr und datierten durch den Blick darauf den Moment, in dem bewusst die Handlung eingeleitet wurde. Woher wissen wir aber, wie viel Zeit dieser komplexe Prozess der Selbstbeobachtung und die dann notwendige Korrelation mit der äußeren Uhr in Anspruch nimmt? Wir müssen für den Messvorgang irgendwie die Innenperspektive und die Außenperspektive aufeinander abstimmen. Dazu reizte Libet bei den Versuchspersonen die Haut und ließ sie mit Hilfe der erwähnten Uhr berichten, wann sie sich dieser Hautreizung bewusst wurden. Somit konnte der Zeitunterschied zwischen äußerer Reizung und berichteter Uhrzeit bestimmt werden. Aber kann man dadurch sicher sein, dass man auch im Falle der Handbewegung richtig misst? Vielleicht geschieht die bewusste Wahrnehmung einer äußeren Reizung der Haut schneller als die bewusste Selbstreflexion auf das Bedürfnis, die Hand zu bewegen? Letzteres ist ein künstliches, unnatürliches Verhalten, ersteres ein evolutionär sehr wichtiges Geschehen, um Verletzungen vom Körper fernzuhalten. Neuere Experimente von William Banks scheinen in

\footnotetext{
${ }^{3}$ Libet, Benjamin: Mind Time (dt. Ausgabe). Frankfurt: Suhrkamp, 2005.
} 
der Tat zu belegen, dass die Versuchspersonen den Zeitpunkt der bewussten Entscheidung nicht verlässlich berichten können. Wenn man beispielsweise die Fingerbewegung mit einem Signalton bestätigt und diesen dann absichtlich verzögert, also ein klein wenig nach der Bewegung erklingen lässt, dann datieren die Versuchspersonen ihre bewusste Entscheidung, den Finger zu bewegen, entsprechend der Verzögerung nach vorne. Sie beziehen also das Ergebnis der bewussten Entscheidung in die Bestimmung des Zeitpunktes der bewussten Entscheidung mit ein. ${ }^{4}$ Das ist ein Hinweis darauf, dass die zeitliche Bestimmung eines bewussten Erlebnisses ein komplexer Akt der Konstruktion ist, keine direkte Wahrnehmung. Bei genauem Hinsehen sind die Dinge also nicht mehr so klar. Die ermittelten Daten beruhen in der Regel auf etwa 40 Durchgängen. Bei Libet trat im Mittel das Bereitschaftspotential ca. 500 ms vor der bewussten Einleitung der Bewegung auf. Andere Autoren, die das Experiment wiederholten, fanden zum Teil erheblich abweichende Ergebnisse. Interessanterweise zeigte sich bei einigen Versuchsreihen das Bereitschaftspotential statistisch signifikant häufig auch nach der Bewegung und nicht vorher. ${ }^{5}$ Ein wesentlicher Gesichtspunkt ist hier, dass die bewusste Aufmerksamkeit die Zeitwahrnehmung verändert. Je nachdem, ob die Person sich mehr auf den optischen Reiz der Uhr oder auf die bewusste Selbstwahrnehmung konzentriert hat, können unterschiedliche subjektive Datierungen berichtet werden. Naturwissenschaftliche Experimente müssen daher immer sehr genau analysiert werden. Die trivialen Darstellungen in den populären Medien führen nicht selten zu Halbwahrheiten. Trotzdem kann man sagen, dass heute eine sehr starke empirische Evidenz die Existenz der beschriebenen Bereitschaftspotenziale und ihre Wirksamkeit bestätigt. Viele Körperbewegungen werden also schon in einem unbewussten Prozess initiiert, das Bewusstsein wird erst zu einem späteren Zeitpunkt als Kontrollinstanz eingeschaltet.

Wenn aber ein wesentliches Charakteristikum der Willensfreiheit darin besteht, Kontrolle über die eigenen Bestrebungen und Regungen zu haben, also ihre Wirksamkeit für

\footnotetext{
${ }^{4}$ Banks, William, et al.: "We infer rather than perceive the moment of decision to act in Libet's measurement of the time of conscious decision". Towards a science of consciousness. Research abstracts. (2008): 69f.

${ }^{5}$ Keller, I. / Heckhausen, H. "Readiness Potentials Preceding Spontaneous Motor Acts: Voluntary vs. Involuntary Control." Electroencephalography and Clinical Neurophysiology 76 (1990): 351-61.

Haggard, P. / Eimer, M. "On the Relation Between Brain Potentials and the Awareness of Voluntary Movements." Experimental Brain Research 126 (1999): 128-33.
} 
Handlungen beeinflussen zu können, dann kann man nicht erkennen, wie derartige Experimente überhaupt gegen Willensfreiheit sprechen sollen. Es ist nämlich so, und das wurde auch durch neuere Experimente bestätigt, dass die handelnde Person sich gegen das Bereitschaftspotenzial entscheiden und damit gleichsam ein Veto einlegen kann. ${ }^{6}$ Allein das ist schon eine Form von Freiheit. Die neueren Experimente hatten beispielsweise folgenden Aufbau: Die Probanden befanden sich vor einem Bildschirm, der abwechselnd die Farben Grün und Rot darstellte. Wenn der Bildschirm grün war, sollten die Teilnehmerinnen mit dem Fuß ein Pedal bedienen, in einer roten Phase hingegen nicht. Die Hirnsignale der Probanden wurden in einen Computer eingespeist. Dieser Rechner lernte das Muster zu erkennen, dass sich kurz vor einer Fußbewegung aufbaute. Der Computer konnte somit erkennen, wann das Bereitschaftspotenzial für das Drücken mit dem Fuß gegeben war. Dieses Bereitschaftspotenzial liegt bereits vor der bewussten Wahrnehmung der kurz bevorstehenden Fußbewegung messbar vor. Wann immer ein solches Bereitschaftspotenzial festgestellt wurde, schaltete in einer zweiten Phase des Experiments der Computer den Bildschirm auf Rot. Die Versuchspersonen mussten nun also schnell die unbewusst bereits getroffene Entscheidung korrigieren und nicht auf das Pedal drücken. Obwohl die Bewegung mit dem Fuß bereits in Vorbereitung war, musste diese nun durch ein willentliches Veto abrupt gestoppt werden. Dies gelang nicht immer, aber doch in einem erheblichen Umfang. Allein dies reicht völlig aus, um zu zeigen, dass die bewussten Willensentscheidungen immer noch Kontrolle ausüben konnten. Wir können eine unbewusst eingeleitete Bewegung noch durch ein bewusstes Veto kontrollieren. Allerdings, und das kann nicht überraschen, gab es einen „point of no return“, nach welchem die bewusste Entscheidung einfach zu spät kam, um die bereits eingeleitete Bewegung noch aufzuhalten. Die Kontrolle des bewussten Willens ist also nicht absolut, was ohnehin vernünftigerweise nicht zu erwarten war. Der empirische Befund widerlegt also nicht Freiheit im Sinne bewusster Selbstkontrolle.

\section{Freiheit falsch verortet}

${ }^{6}$ Schultze-Kraft, Matthias; Birman, Daniel; Rusconi, Marco; Allefeld, Carsten; Görgen, Kai; Dähne, Sven; Blankertz, Benjamin; Haynes, John-Dylan (2016-01-26). "The point of no return in vetoing self-initiated movements." Proceedings of the National Academy of

Sciences. 113 (4): 1080-1085. 
Ein anderer Gesichtspunkt ist für die philosophische Analyse der fraglichen Experimente besonders wichtig. Es soll im Folgenden wiederum am klassischen Experiment von Libet verdeutlicht werden. Die Versuchsperson hatte hier gar keinen Grund, den Finger in der, sagen wir, 15. statt in der 18. Sekunde zu heben. Sie wartete darauf, dass in der leicht angespannten Situation des voranschreitenden Sekundenzeigers in ihr ein Bedürfnis spontan hervorsprudelte, den Finger zu bewegen. Die Anweisungen für die Probanden sahen vor, dass diese sich nicht einen bestimmten Zeitpunkt für die Bewegung auswählen, sondern den Impuls zur Bewegung spontan in sich aufsteigen zu lassen. Eine klare Willensentscheidung liegt hier gerade nicht vor. Eine Willensentscheidung läge vor, wenn ich zwischen zwei Handlungsalternativen die Gründe abwägen würde, um dann zu einer verantworteten Entscheidung zu kommen. Ein Beispiel: Wenn ich am Morgen vor einem wichtigen Termin im Bett liege und gerade von meinem Wecker geweckt wurde, dann kann ich mir vielleicht sagen: „Ich habe noch fünf Minuten, dann muss ich aufstehen wegen des wichtigen Termins“. Wenn ich mich dann kurze Zeit später spontan und ruckartig aus dem Bett erhebe, weil ein entsprechender Drang in mir aufsteigt, dann ist diese Handlung nicht dadurch unfrei, dass sie halbautomatisch und wie von selbst vor sich geht. Meine Freiheit bestand darin, mich überhaupt für den frühen Termin und das damit verbundene frühe Aufstehen aus Gründen heraus zu entscheiden. Ebenso bestand die Freiheit der Teilnehmer an dem oben beschriebenen Experiment vor allem darin, sich überhaupt als Testperson zur Verfügung zu stellen. Dabei haben sie sicher Gründe für und wider abgewogen. Die kleine Fingerbewegung im Versuch ist hingegen überhaupt kein interessanter Fall für die Erforschung des freien Willens. Es ist nicht bedeutsam, wenn sie weitgehend automatisch abläuft. Sie ist vergleichbar dem automatischen Start der oben erwähnten Läuferin oder auch den automatisierten Vorgängen beim Autofahren. Die Freiheit ist in dem größeren Handlungskontext zu verordnen, nicht in den untergeordneten Prozessen, die gar nicht rational abgewogen werden.

Wenn uns die Psychologie also zu Recht darauf hinweist, dass viele unserer Handlungen und Aktivitäten mindestens teilweise automatisch ablaufen, dann ist das noch keine Widerlegung der Freiheit. Die bisherigen psychologischen Experimente zeigen uns nur, dass viele unserer Aktivitäten ohne unsere bewusste Kontrolle ablaufen. Daraus folgt aber noch nicht, dass alle unsere Handlungen vollständig vorherbestimmt sind durch Ereignisse, die außerhalb unserer bewussten Kontrolle liegen. Wer die Freiheit leugnen will, muss zeigen, dass für sämtliche 
menschliche Handlungen gilt, dass sie von etwas verursacht wurden, das außerhalb der Kontrolle der handelnden Personen liegt. Diese These reicht weit über den Bereich der Psychologie hinaus. Es ist die metaphysische These des universellen Determinismus. Es ist also die Annahme des Determinismus, die eigentlich im Hintergrund fast aller Leugnungen der Willensfreiheit steht. Wenn das Gehirn zu jedem beliebigen Zeitpunkt nur eine mögliche Zukunft hat und wenn unsere Handlungen vom Gehirn abhängen, dann werden unsere Handlungen letztlich bestimmt von Zuständen im Gehirn, die weit in unserer Vergangenheit zurückliegen. Letztlich lassen sie sich aus einer entfernten Vergangenheit ableiten, in der wir noch gar nicht über die Möglichkeit der willentlichen Selbstbestimmung verfügten. Aus einem frühen Gehirnzustand, der von Genen und Umwelteinflüssen determiniert ist, folgen mit naturgesetzlicher Notwendigkeit alle anderen späteren Gehirnzustände. Die These soll nun sein, dass in einer solchen deterministischen Welt Freiheit nicht möglich ist.

\section{Zurück zur Philosophie}

Die Ergebnisse der empirischen Forschung haben uns also wieder zurückgeführt zu unseren philosophischen Überlegungen. Es sind nicht primär die empirischen Ergebnisse, die zur Leugnung der Freiheit führen, sondern die starke metaphysische These des universellen Determinismus. An dieser Stelle wird klar, warum wir mit einer philosophischen Analyse des Freiheitsbegriffes begonnen hatten. Wir hatten nämlich weiter oben bereits gezeigt, dass es durchaus einen gehaltvollen Begriff der Willensfreiheit gibt, der mit der Annahme des universellen Determinismus vollkommen verträglich ist. Wie bereits dargelegt, erlaubt es die kompatibilistische Konzeption, freie von unfreien Handlungen in einer deterministischen Welt zu unterscheiden. Freiheit ist dann gegeben, wenn die handelnde Person sich nach einem bewussten Abwägungsprozess, der die Fähigkeit zur Unterscheidung von moralisch Gutem vom moralisch Schlechten einbezieht, weder durch inneren psychischen noch durch äußeren Zwang daran gehindert sieht, ihre Wünsche in die Tat umzusetzen. Diese Art von Freiheit wird durch Determination nicht beeinträchtigt. Selbst komplexe Fälle innerer Unfreiheit können in diesem Freiheitskonzept überzeugend rekonstruiert werden. Ein Mensch hat Freiheit des Willens nur dann, wenn er den inneren Strebungen nicht einfach ausgeliefert ist wie ein unmündiges Kind. Stellen wir uns eine drogensüchtige Person vor, die über die ersehnte Droge verfügt. Als abhängige Person wird sie in sich ein Begehren fühlen, 
die Droge jetzt auch zu konsumieren. Stünde es fest, dass die Person in dieser Lage automatisch und ohne Ausnahme die Droge konsumierte, dann wäre sie nicht frei, sondern in einem zwanghaften Mechanismus gefangen. Freiheit des Willens kommt genau dann ins Spiel, wenn sich der Drogenabhängige noch fragen kann: „Will ich die Droge wirklich konsumieren?“ Wenn er dann Willenskraft mobilisieren und sich innerlich sagen kann „Eigentlich will ich gar nicht, was ich begehre, weil es mir schadet!“, dann tritt ein Moment der Selbstkontrolle auf die geistige Bühne. Aber selbst gesunde Erwachsene verfügen oft nur eingeschränkt über die Fähigkeit, ihre eigenen Bedürfnisse zu prüfen und zu kontrollieren. Derartige Willensschwäche ist eine Beschränkung unserer Freiheit. Die willensschwache Person tut Dinge, die sie eigentlich nicht tun will, oder sie unterlässt Dinge, die sie eigentlich tun will. Dieser Mangel an Selbstbestimmung ist zugleich ein Verlust an innerer Freiheit. Das eigentliche Problem der Willensschwäche liegt nicht darin, dass wir manchmal unbeherrscht sind. Es liegt darin, dass die willensschwache Person sich nicht rational verhält. Sie handelt wider bessere Einsicht. Wir wissen zum Beispiel, dass es ungesund ist, bestimmte Lebensmittel zu essen. Trotzdem tun wir es immer wieder. Es ist nicht unvernünftig, ganz gelegentlich mal eine spezielle Ausnahme zu machen. Irrational verhält man sich, wenn man langfristig und wiederholt etwas tut, dass man eigentlich nicht will. Dieser Fall ist keineswegs allein auf die Sucht beschränkt. Wir alle kennen das Phänomen, dass man schleichend nach dem Motto „einmal ist keinmal“ immer tiefer in eine Gewohnheit gerät, die den eigenen besten Einsichten entgegenläuft. Im juristischen Sinne ist eine Person, die einen solchen schleichenden Prozess der Unterminierung der eigenen Autonomie zugelassen hat, selbstverständlich dafür auch verantwortlich. Als rationales Wesen war ihr die Vernunftwidrigkeit ihres Handelns vollständig bewusst. Aber dennoch ist zu konstatieren, das am Ende dieses Prozesses eine Verminderung der Autonomie und Willensfreiheit steht. Wirkliche Freiheit hat man erst dann erworben, wenn man sich über seine Lebensziele und Projekte im Klaren ist und auch die Kraft hat, sich nachhaltig und motiviert für diese einzusetzen.

Hier sieht man einen deutlichen Unterschied zwischen Freiheit und Zufall. Der freie Mensch schwankt nicht zufällig zwischen dieser und jener Handlung hin und her, sondern er ordnet sein ganzes Handeln mit praktischer Vernunft auf die Ziele aus, die er sich gesetzt hat. All dies lässt sich unter der Annahme einer deterministischen Welt behaupten. Welches Problem sollte also durch die Annahme des Determinismus aufgeworfen werden? 
Im Libertarismus lassen sich alle diese Überlegungen zur willentlichen Selbstbestimmung direkt übernehmen. Der relevante Unterschied ist die zusätzliche Annahme einer offenen Zukunft. Diese Annahme ist nicht wirklich problematisch. Wir wissen nämlich zum gegenwärtigen Zeitpunkt nicht mit Gewissheit, ob unsere Welt nach ausnahmslos strikten kausalen Sukzessionsgesetzen abläuft, oder ob nicht doch ein Raum für eine offene Zukunft gegeben ist. Nehmen wir einmal eine offene Zukunft an. Nehmen wir dann auch noch an, dass unsere bewussten Entscheidungen eine kausale Kraft auf die Welt ausüben können, dass es also mentale Verursachung von Handlungen gibt. Dann könnte ein Indeterminismus im Bereich der mentalen Zustände den Raum für mehrere mögliche Handlungen eröffnen, die mit ein und derselben Vergangenheit verträglich sind. Es gibt dann für die handelnde Person mehr als eine mögliche Zukunft. Wenn dies der Fall ist, dann steht nicht alles, was sich heute ereignet seit unvordenklichen Zeiten bereits fest. Die handelnde Person selbst bestimmt mit, welche mögliche Zukunft real wird. Man kann mit guten Gründen eine solche offene Zukunft für eine notwendige aber nicht hinreichende Bedingung von Freiheit halten. Wirkliche Verantwortung für eine Handlung hat nur jemand, der auch anders hätte handeln können. Keines der oben dargestellten Experimente in den empirischen Wissenschaften zwingt uns dazu, diese indeterministische Sicht der Welt aufzugeben. Auch in einer solchen libertarischen Konzeption liegen die empirischen Kriterien einer freien Handlung darin, dass sie bewusst aus durch die Vernunft erkannten Gründen und ohne Zwang erfolgt. Hier gibt es keinen Unterschied zum Kompatibilismus. Entscheidend sind also bestimmte Fähigkeiten, die wir einer freien Person zuschreiben: Vernunftgebrauch, Selbstkontrolle, Bewusstsein. Die geforderten Fähigkeiten sind in beiden großen Konzeptionen der Willensfreiheit nahezu dieselben.

Es sind dies auch dieselben Fähigkeiten, die auch der gesunde Menschenverstand für Willensfreiheit fordert. Was sagen Durchschnittsbürger mit gesundem Menschenverstand, wenn man sie danach fragt, was Freiheit ausmacht? Am einfachsten erhält man eine Antwort, wenn man die Befragten bittet, Ereignisse aus ihrem Leben zu beschreiben, in denen sie den Eindruck hatten, sich frei zu entscheiden. Kontrastierend kann man sie bitten, auch solche Erlebnisse zu berichten, in denen sie sich gerade nicht als frei erlebten. ${ }^{7}$ Frei erleben sich Menschen besonders in Situationen, in denen sie sich mit langfristigen

\footnotetext{
${ }^{7}$ Stillman, T. F., Baumeister, R. F., \& Mele, A. R. „Free will in everyday life: Autobiographical accounts of free and unfree actions." Philosophical Psychology 24 (2011): 381-394.
} 
zukünftigen Zielen und Projekten auseinandersetzen. Frei erleben sie Handlungen, die mit ihren inneren Werten und Zielen übereinstimmen. Frei erleben sie sich, wenn Sie Zeit haben, über die verschiedenen Alternativen nachzudenken, um sie mit Argumenten gegeneinander abzuwägen. Mehr noch: Jüngere Untersuchungen haben gezeigt, dass die Annahme einer offenen und erst noch zu entscheidenden Zukunft für das alltägliche Verständnis von Willensfreiheit wichtig ist. Menschen verstehen sich selbst nicht als Akteure, die vollkommen von der Vergangenheit, also von ihren vergangenen Wünschen, Überlegungen und Antrieben bestimmt sind. Einige Psychologen haben deshalb argumentiert, dass sich ihre Wissenschaft vom „driven by the past“ Modell verabschieden solle. ${ }^{8}$ Freiheit ist dann nicht nur als die Abwesenheit von Zwang zu verstehen. Wir erfahren uns als frei, wenn wir mehrere mögliche Verläufe der Zukunft beschreiben und evaluieren können und unsere Handlungen als ein Ergebnis dieses Prozesses auswählen können. Das libertarische Modell der Willensfreiheit, für das die Offenheit der Zukunft zentral ist, liegt daher näher am Selbstverständnis der meisten Menschen. Das wissenschaftliche Weltbild hingegen insistiert auf der Bedeutung der kausalen Bestimmung durch die Vergangenheit. Etwas, das nicht vollständig aus der Vergangenheit erklärt werden kann, entzieht sich damit dem wissenschaftlichen Zugriff.

Von daher kann man wirklich sagen, dass es eine Spannung zwischen dem wissenschaftlichen Weltbild einerseits und dem im „common sense“ verankerten Libertarismus gibt. Wir haben aber versucht aufzuzeigen, dass sich diese Spannung auf zwei verschiedenen Wegen auflösen lässt. Man kann einerseits den alltäglichen Libertarismus aufgeben und eine kompatibilistische Konzeption der Freiheit vertreten. Andererseits ist der universelle Determinismus keine empirische These, die sich aus unseren besten naturwissenschaftlichen Theorien zwingend ergäbe. Man kann also alternativ den Determinismus aufgeben und am Libertarismus festhalten. Eine libertarische Freiheitskonzeption, für die die Annahme von mehr als einer möglichen Zukunft wesentlich ist, steht nicht im logischen Widerspruch zu unserem naturwissenschaftlichen Wissen. Man verhält sich also nicht unwissenschaftlich, wenn man der Alltagsintuition folgt, dass sich Freiheit vor allem als rationale Selbstbestimmung angesichts mehrerer möglicher Zukunftsverläufe verstehen lässt.

\footnotetext{
${ }^{8}$ Martin E. P. Seligman, Peter Railton, Roy F. Baumeister and Chandra Sripada. "Navigating Into the Future or Driven by the Past." Perspectives on Psychological Science 8 (2013): 119.
} 


\section{Schlussbetrachtung}

Unsere philosophischen Analysen haben also ergeben, dass sich zwei in sich stimmige Konzeptionen der Freiheit entwickeln lassen. Freiheit wird also nicht zwischen Notwendigkeit und Zufall zerrieben. Es wurde auch offensichtlich, dass keine der beiden großen Freiheitskonzeptionen, Kompatibilismus und Libertarismus, eindeutig die argumentative Oberhand über die Alternative erringen konnte. Wie bei vielen anderen philosophischen Problemen findet man keine "Lösung", die allen anderen deutlich überlegen ist. Im weiteren Verlauf wurde versucht aufzuzeigen, dass empirische Untersuchungen und Experimente bisher nicht in der Lage waren, die Existenz von Willensfreiheit schlüssig zu widerlegen. Wenn Wissenschaftler einen Konflikt zwischen Freiheit und Wissenschaft erkennen, dann meistens deshalb, weil sie eine alltägliche libertarische Konzeption der Freiheit vertreten und diese mit der deterministischen Weltsicht nicht in Einklang bringen können, die sie der Naturwissenschaft zu Grunde legen wollen. Wir haben versucht aufzeigen, dass dieser Konflikt entschärft werden kann.

Für die juristische Praxis kann nun mit guten Gründen die Übernahme einer allgemeinen Liste von Fähigkeiten und Bedingungen empfohlen werden, die freien Akteuren zukommen. Diese Liste soll nur Fähigkeiten und Bedingungen enthalten, die von Kompatibilisten und Libertariern gleichermaßen als relevant anerkannt werden können. Damit ist die größtmögliche Akzeptanz garantiert. Folgende Liste erfüllt diese Anforderungen:

(1) Freie Akteure handeln aus Gründen. Ihr Verstand erfasst intentional einen Begründungszusammenhang.

(2) Freie Akteure müssen in der Lage sein, normative Zusammenhänge zu erfassen.

(3) Bei freien Akteuren muss der Prozess, der von einer freien Entscheidung zu einer freien Tat führt, nicht durchweg bewusst ablaufen, aber sich in einem übergeordneten Kontext bewusster Abwägung von Gründen befinden.

(4) Freie Akteure dürfen nicht durch äußeren Zwang an der Ausführung ihrer Handlungsabsichten wirksam gehindert werden.

(5) Freie Akteure dürfen nicht durch inneren psychischen Zwang an der Ausführung ihrer Handlungsabsichten wirksam gehindert werden.

Gegen einen Freiheitsbegriff, der sich an diesen 5 Kriterien orientiert, gibt es weder durchschlagende empirische Evidenz noch unausweichliche philosophische Argumente. Für die Praxis der Rechtsprechung reicht diese Bestimmung von Freiheit vollständig aus. 
Juristen und Juristinnen sollten sich also trotz der aktuell populären Zweifel nicht scheuen, den Freiheitsbegriff weiterhin zu verwenden. Der gesunde Menschenverstand würde den Verzicht darauf mit Recht nicht nachvollziehen können. Der Freiheitsbegriff ist für den Menschen schlechterdings zentral. Er ist auch aus dem Rechtswesen nicht wegzudenken. 